\title{
Friction of Teflon-S-coated Ti-6Al-4V under conditions of oscillatory relative motion
}

\author{
D. J. Ligterink, G. J. Verkerke and A. W. J. de Gee*
}

An extendable prosthesis for implantation in a human leg has been developed. The friction forces during extension of the prosthesis must be low, so a coating of Teflon-S was applied to the sliding surfaces. During walking, damage can occur as a result of oscillatory relative motion. Therefore experiments were performed with an apparatus which simulated this type of motion. It was found that the coefficient of friction reached a maximum value within one million movement cycles. This maximum value of the coefficient of friction increased with decreasing coating thickness. Even at an initial coating thickness of less than $1 \mu \mathrm{m}$, coating with Teflon-S was found to be very effective in reducing friction.

Keywords: oscillatory relative motion, extendable prosthesis, friction coefficient, Teflon-S

\section{Introduction}

A bone tumour may develop around the knee joint of a child. In most cases it will then be necessary to resect the bone involved including the growth plates, knee joint and adjacent tissue. A joint team from the Gröningen University Hospital and the University of Twente has developed a modular endoprosthetic system to bridge the defect resulting from the resection. Since the other, normal leg continues to grow, the endoprosthetic system includes a prosthesis, the length of which can be adjusted without an operation. It is to be expected that after one or two months a lengthening of $2 \mathrm{~mm}$ must be performed. ${ }^{1,2}$

The extendable prosthesis is telescopic, consisting of two tubes, with one sliding into the other (Fig 1). Relative rotation is prevented by making the tubes polygonal in shape. The extendable prosthesis is activated by an external rotating magnetic field causing rotation of a small permanent magnet in the prosthesis. The magnet drives a motion screw via a gearbox. The motion screw rotates in the outer tube and forces the two telescopic tubes apart. In order to shield the extendable prosthesis from moisture a bellows, made of silicone rubber, is glued to the prosthesis. The tubes and motion screw are made from Ti-6Al- $4 \mathrm{~V}$, one of the few alloys suitable for implantation. It is strong and is easy to machine. The drawback of having two adjacent Ti-6Al-4V surfaces is that such contacts usually suffer from high friction and severe adhesive material transfer. To decrease the friction losses in the prosthesis, caused by the rotating motion screw and

* University of Twente, Faculty of Mechanical Engineering, P.O. Box 217, 7500 AE Enschede, The Netherlands
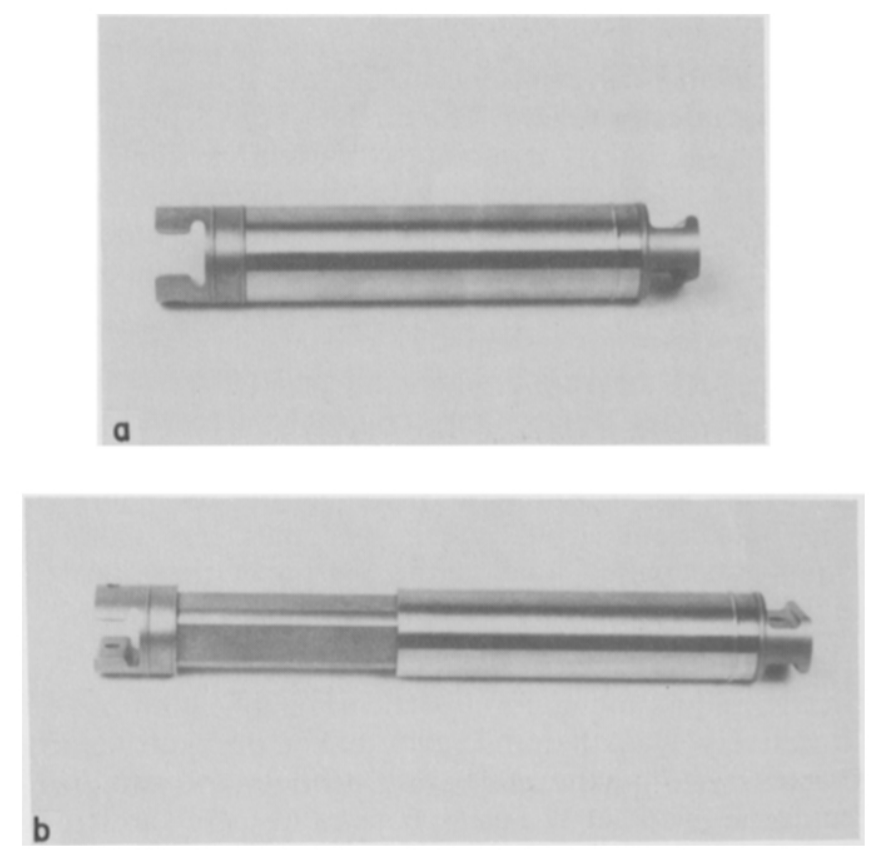

Fig 1 Extendable prosthesis (without bellows) (a) before extension and (b) after full extension

the sliding telescopic tubes, both the outer tube and the motion screw were coated with Teflon-S (Fig 2).

During walking both the motion screw and the telescopic tubes will be subjected to variable forces and, probably, small relative movements which may damage the coating. The load on the coated surface can lead to a pressure up to $100 \mathrm{MPa}$. The maximum amplitude of the movement cycle is of the order of 

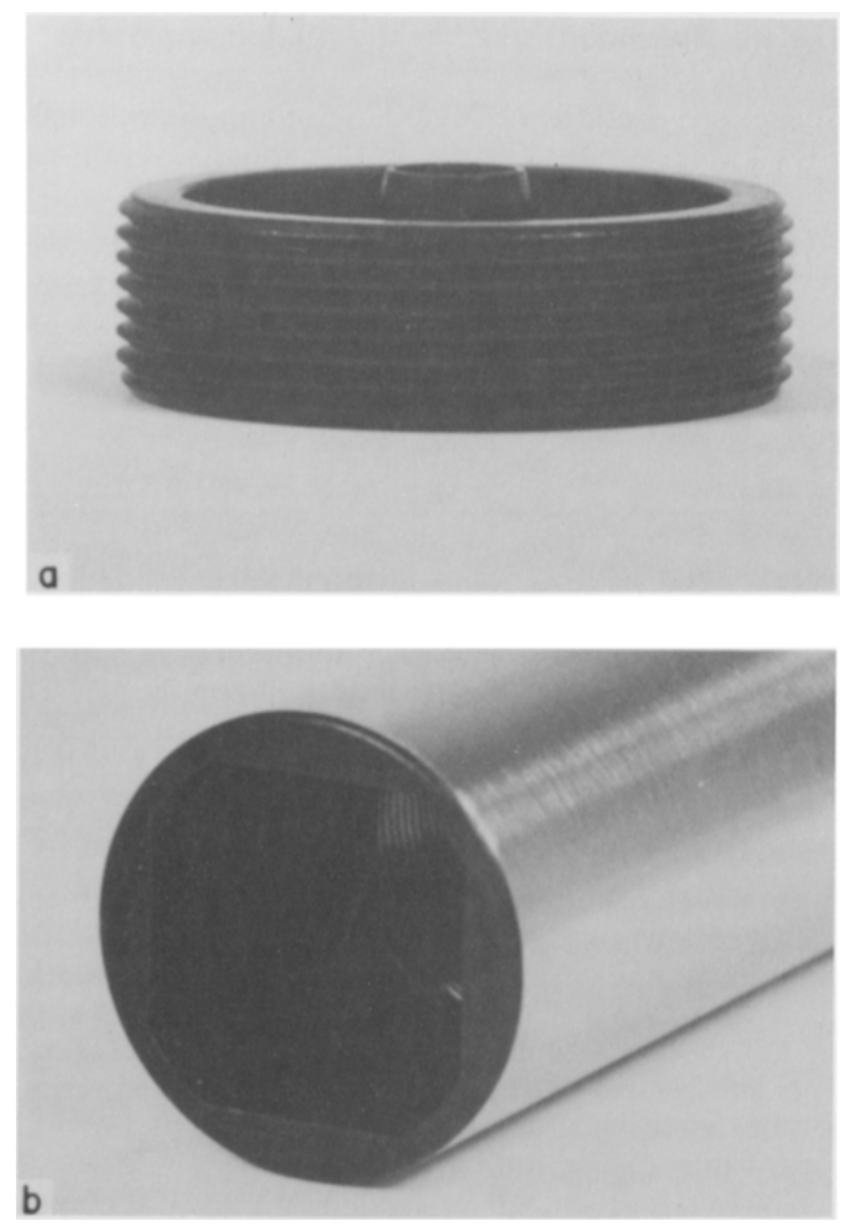

Fig 2 (a) Coated screw, diameter $=27 \mathrm{~mm}$; (b) polygonal tube, diameter $=30 \mathrm{~mm}$

$100 \mu \mathrm{m}$. As the Teflon-S coating is expected to be essential for a good performance of the prosthesis, it was considered necessary to determine the intensity of possible damage, particularly with respect to friction. A high value of the coefficient of friction $(f \approx 0.3)$ would lead to an unacceptable increase in the force necessary for extending the prosthesis. ${ }^{3}$

\section{Experimental details}

\section{Specimen shape and initial pressures}

In the screw, contact between nominally flat surfaces occurs. As it is virtually impossible to ensure full conformity between two flat surfaces (in practice as well as in the laboratory), a sphere in contact with a flat surface was chosen for the experiments. In such a contact application of a normal force $F_{\mathrm{n}}$ will cause elastic deformation, with an initial maximum pressure $p_{\max }$ as determined by the well-known Hertzian equations.

Given values of $p_{\max }$, the radius $R$ of the sphere that is to be applied relates to normal force $F_{\mathrm{n}}$ as $^{4}$

$$
R^{2}=0.365^{3} E^{2}\left(F_{\mathrm{n}} / p_{\text {max }}^{3}\right)
$$

in which $E$ is given by

$$
E=\frac{2 E_{1} E_{2}}{\left(1-v_{2}^{2}\right) E_{1}+\left(1-v_{1}^{2}\right) E_{2}}
$$

$E_{1}$ and $E_{2}$ are the elastic moduli and $\nu_{1}$ and $\nu_{2}$ the Poisson constants of the contacting surfaces 1 and 2 , respectively. In the present case the sphere is made from uncoated titanium alloy, while the flat surface is coated with Teflon-S. As the coatings are thin (maximum $20 \mu \mathrm{m}$ ) and probably become even thinner upon the application of normal force, it is assumed that they do not contribute significantly to load carrying capacity. Thus in this case we take $E_{1}=E_{2}=$ $E_{\mathrm{T} \text {-alloy }}=115 \mathrm{GPa}$ and $v_{1}=v_{2}=0.3$.

As stated above the apparent contact pressure $p$ in the tube amounts to approximately $100 \mathrm{MPa}$. As the system should also function adequately if misalignment and, thus, a higher contact pressure occurs, a contact pressure three times higher $\left(p_{\max }=300 \mathrm{MPa}\right)$ was chosen in the present experiments. Taking $F_{\mathrm{n}}=7 \mathrm{~N}$, a convenient value from an experimental point of view, Eq 1 yields an $R$ value of $13 \mathrm{~mm}$. This value was applied in all experiments.

\section{Apparatus}

The experiments were performed with a newly constructed apparatus, shown (schematically) in Figs 3 and 4. A particular advantage of this apparatus is that the electro-magnetic excitation of the driving rod causes a minimum of mechanical distortions, which might influence the measurement signals. A disadvantage is that during an experiment the amplitude of the movement tends to decrease, because of increasing friction. Thus it has to be adjusted regularly during an experiment (allowing a decrease in amplitude of maximum $5 \%$ of the initial value). The experiments were performed with an amplitude of $50 \mu \mathrm{m}$ and frequencies of $10 \mathrm{~s}^{-1}$ or $20 \mathrm{~s}^{-1}$, chosen arbitrarily. The environment of the sliding surfaces in the prosthesis will be dry or wet (condensed water film). As dry

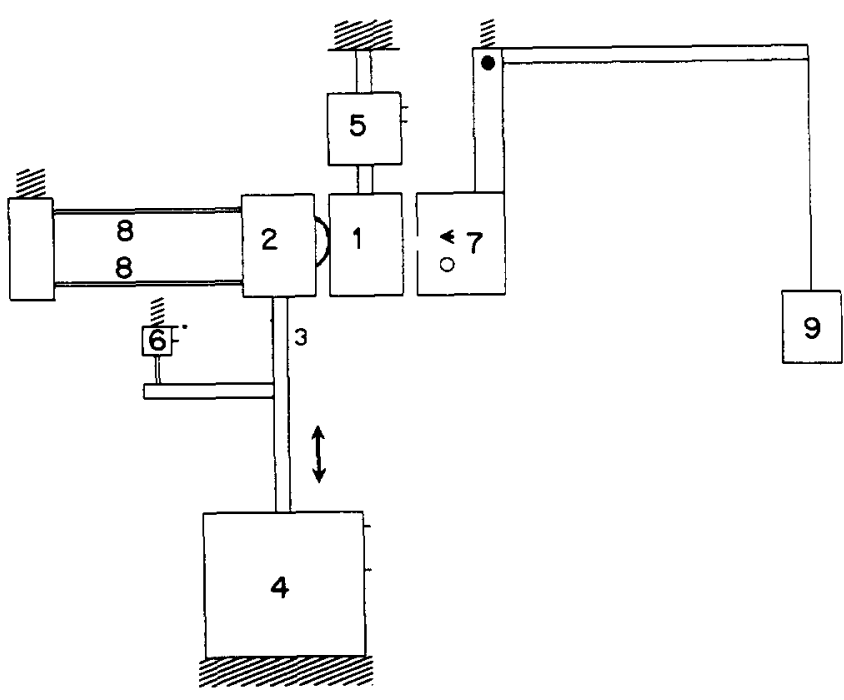

Fig 3 Diagram of the friction apparatus: 1: flat specimen (coated); 2: spherical specimen (titanium alloy); 3: driving rod; 4: exciter (electro-magnetic; controlled by function generator); 5: force transducer (quartz); 6: displacement transducer (electromagnetic); 7: introduction normal force (aerostatic); 8: springs; 9: load; $\equiv$ : earth, $\bigcirc$ : air inlet 


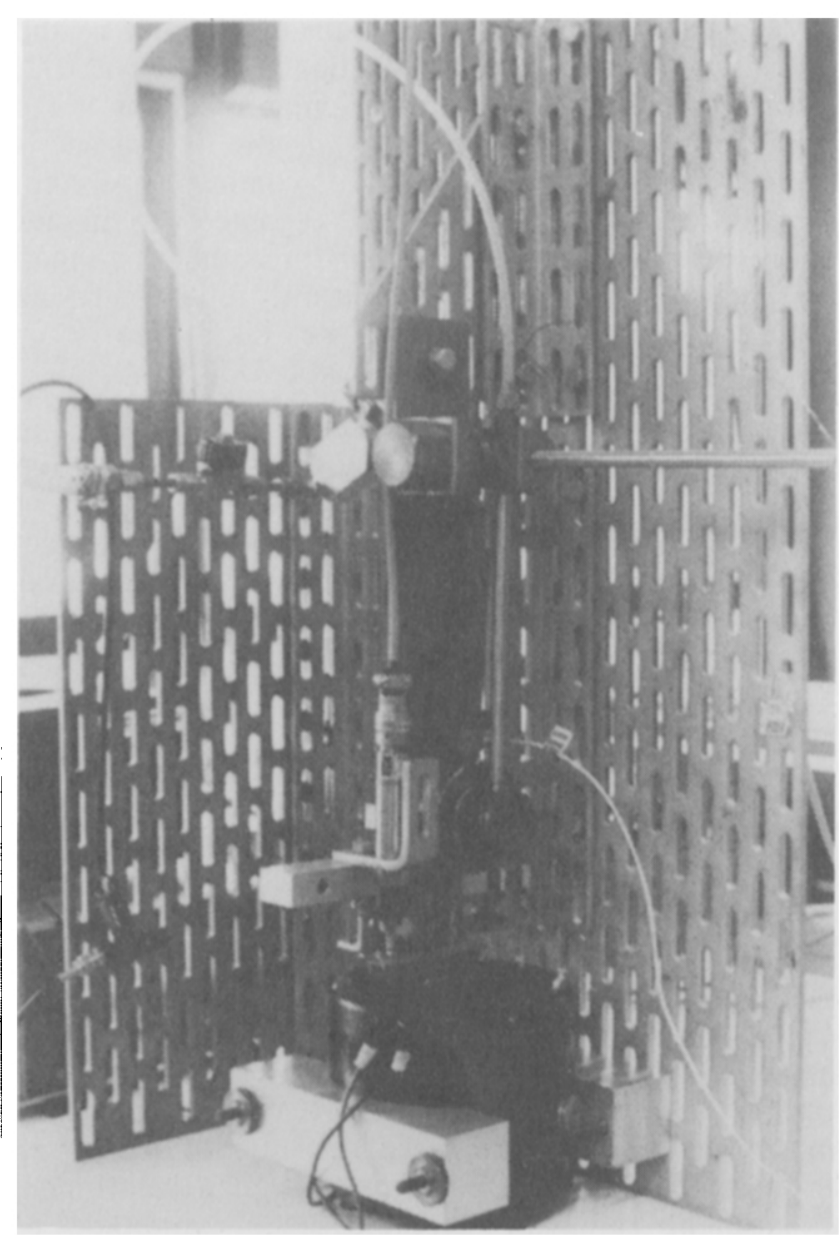

Fig 4 Friction apparatus

contact is probably the most unfavourable, air was chosen as the experimental environment.

\section{Materials}

Fig 5 shows a specimen couple: In Table 1 the specimens are described in some detail. The spherical specimens 1,3 and 5 were of uncoated titanium alloy. Their surface roughness $\left(R_{1}\right.$, peak-to-valley value) amounted to approximately $1 \mu \mathrm{m}$. The flat specimens

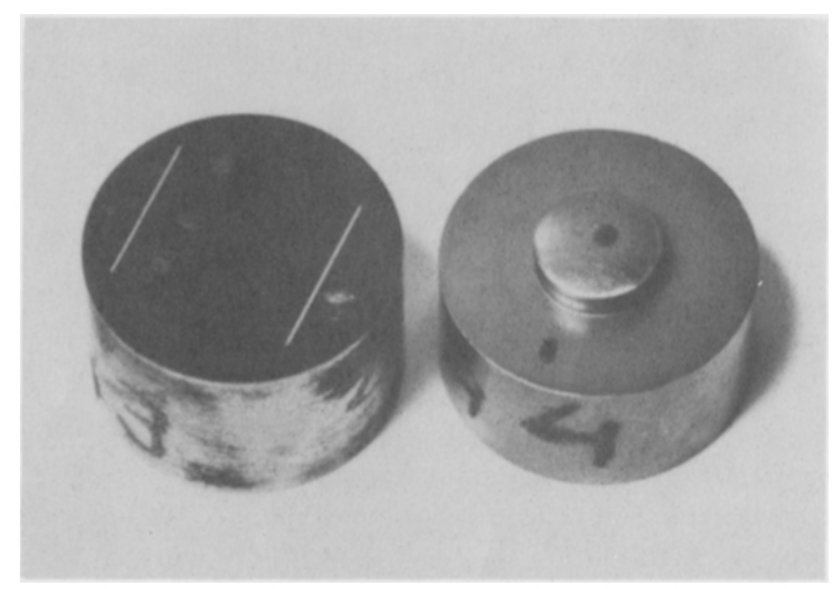

Fig 5 A couple of specimens: diameter $=15 \mathrm{~mm}$, height $=10 \mathrm{~mm}$
Table 1 Specimens used in the friction apparatus

\begin{tabular}{lcccc}
\hline $\begin{array}{c}\text { Specimen } \\
\text { Radius, } \\
\mathrm{mm}\end{array}$ & $\begin{array}{c}\text { Coated } \\
\end{array}$ & $\begin{array}{c}\text { Coating Roughness, } \\
\text { thickness, } \\
\mu \mathrm{m}\end{array}$ & \\
\hline 1 & 13 & No & - & $<1$ \\
2 & $\infty$ & Yes & $<1$ & $\approx 17$ \\
3 & 13 & No & - & $\approx 1$ \\
4 & $\infty$ & Yes & 1 to 3 & $\approx 21$ \\
5 & 13 & No & - & $\approx 1$ \\
6 & $\infty$ & Yes & 17 to 28 & $\approx 10$ \\
\hline
\end{tabular}

were coated with Teflon-S in three thickness ranges: less than $1 \mu \mathrm{m}$ (specimen 2); 1 to $3 \mu \mathrm{m}$ (specimen 4); 17 to $28 \mu \mathrm{m}$ (specimen 6). The differences in surface roughness of the coated surfaces depend on the surface roughness of the titanium surface, prior to coating, and on the thickness of the coating. In general a relatively thick coating tends to mask an initially high surface roughness.

In total nine experiments were performed. A spherical specimen was used three times, without changing its position in the specimen holder. Each spot on the coated counter-surface was used only once. Thus each coated specimen supplied three contact spots (Fig 5). The number of movement cycles between two screw settings is to be around $10^{6}$. In experiments $\mathrm{A}$ to $\mathrm{C}$ (see Table 2) the total amount of cycles was set at $1.7 \times 10^{6}$ (frequency $f r=20 \mathrm{~s}^{-1}$ ) and in experiments $D$ to $I$ at $1.05 \times 10^{6}$ (frequency fr $10 \mathrm{~s}^{-1}$ ). This corresponds to an experiment duration of 24 and $29 \mathrm{~h}$ respectively.

\section{Results}

Numerical results, expressed in terms of start value of the coefficient of friction $f\left(f_{n=0}\right)$ and maximum value of $f\left(f_{\max }\right)$ are given in Table 2. These $f$-values compare favourably with those of uncoated titanium alloy upon itself, which amount to approximately 0.8 (Ref 5). Fig 6 shows a recording of the friction force $F_{\mathrm{fr}}$ as a function of the number of cycles $n$ for experiment $\mathrm{E}$ (coating thickness 1 to $3 \mu \mathrm{m}$ ). Fig 7 shows similar information for experiment $\mathrm{H}$ (coating thickness 17 to $28 \mu \mathrm{m})$. It can be seen that in the case of the thin

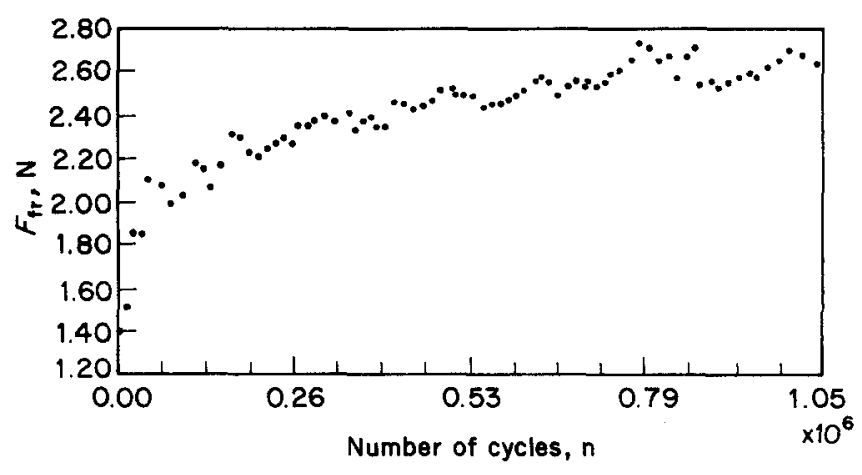

Fig 6 Experiment E: frictional force $\left(F_{\mathrm{fr}}\right)$ as a function of number of cycles, $n$; frequency $=10 \mathrm{~s}^{-1} ; f_{n=0}=0.19$; $f_{\max }=0.38$; coating thickness $=1$ to $3 \mu \mathrm{m}$ 
Table 2 Results of the experiments

\begin{tabular}{lccccc}
\hline Experiment* & $\begin{array}{c}\text { Combination of } \\
\text { specimens** }\end{array}$ & $\begin{array}{c}\text { Coating } \\
\text { thickness, } \mu \mathrm{m}\end{array}$ & $\begin{array}{c}\text { Number of } \\
\text { cycles, } n\end{array}$ & Coefficient of friction \\
\hline A & $1-2.1$ & $<1$ & $1.7 \times 10^{6}$ & $f_{n=0}$ & $f_{\max }$ \\
B & $1-2.2$ & $<1$ & $1.7 \times 10^{6}$ & 0.18 & 0.32 \\
C & $1-2.3$ & $<1$ & $1.7 \times 10^{6}$ & 0.16 & 0.43 \\
D & $3-4.1$ & 1 to 3 & $1.05 \times 10^{6}$ & 0.22 & 0.45 \\
E & $3-4.2$ & 1 to 3 & $1.05 \times 10^{6}$ & 0.19 & 0.31 \\
F & $3-4.3$ & 1 to 3 & $1.05 \times 10^{6}$ & 0.23 & 0.38 \\
G & $5-6.1$ & 17 to 28 & $1.05 \times 10^{6}$ & 0.17 & 0.38 \\
H & $5-6.2$ & 17 to 28 & $1.05 \times 10^{6}$ & 0.18 & 0.30 \\
I & $5-6.3$ & 17 to 28 & $1.05 \times 10^{6}$ & 0.20 & 0.30 \\
\hline
\end{tabular}

* Experiments $A$ to $C$ and $D$ to $I$ are performed with frequencies of $20 \mathrm{~s}^{-1}$ and $10 \mathrm{~s}^{-1}$ respectively.

** The first and second number refer to the specimens, mentioned in Table 1. The third number refers to the spot on the coated specimen.

coating (Fig 6) the $F_{\mathrm{fr}}-n$ tracing is irregular, with friction force, $F_{\mathrm{fr}}$ reaching a maximum value at $n=$ $0.8 \times 10^{6}$. On the other hand, the $F_{\mathrm{fr}}-n$ tracing obtained with the relatively thick coating (Fig 7) is relatively regular and $F_{\text {fr }}$ attains a maximum value at $n=0.25 \times 10^{6}$. Obviously this difference indicates that the thicker coating is most effective at reducing friction.

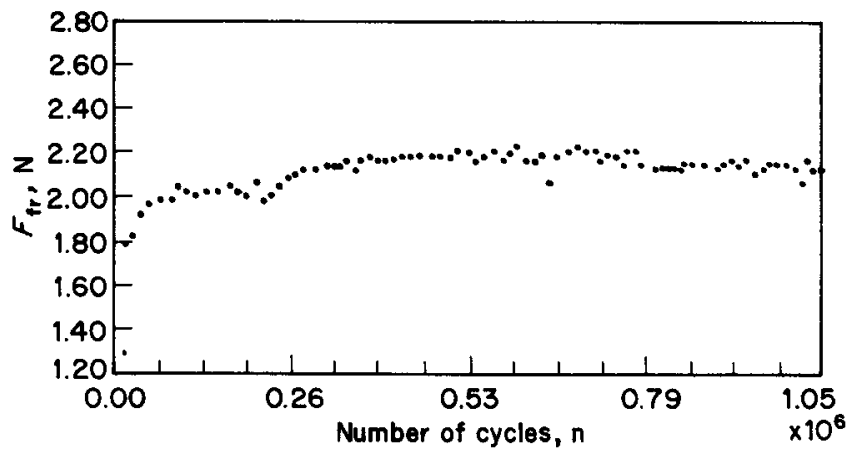

Fig 7 Experiment $H$ : frictional force $\left(F_{\mathrm{fr}}\right)$ as function of number of cycles, $n$; frequency $=10 \mathrm{~s}^{-1} ; f_{n=0}=0.18$; $f_{\max }=0.30 ;$ coating thickness $=17$ to $28 \mu \mathrm{m}$

From the obtained $f$-values the following can be deduced:

- Regarding reduction in friction, coating with Teflon$S$ is extremely beneficial, even in cases where the initial coating thickness is less than $1 \mu \mathrm{m}$.

- Irrespective of the initial coating thickness, initial friction increases with the number of cycles.

- Irrespective of the initial coating thickness, repeated use of a single contact spot on the (uncoated) surface of the sphere leads to an increase of friction at the end of the experiment.

- The maximum value of friction tends to increase with decreasing coating thickness, particularly in the experiments with small coating thicknesses.
Quantitative wear measurements were not performed. However, surface profile measurements, performed after experiment $H$ (Figs 8 and 9 ), reveal that contact with the sphere leads to reduction in coating thickness. Microscopic observation of the contact areas on the coated surfaces after termination of the experiments revealed that in all cases one or more small spots of titanium alloy could be seen. It is likely that a very thin layer of coating material remained attached to the titanium alloy substrate. This is in line with the observations that even a coating with an initial thickness

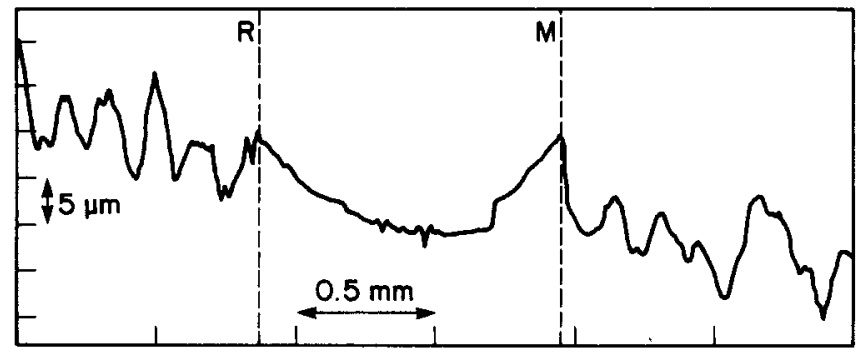

Fig 8 A profile of specimen 6 after experiment $H$, taken parallel to the sliding movement; $R M$ represents the wear spot

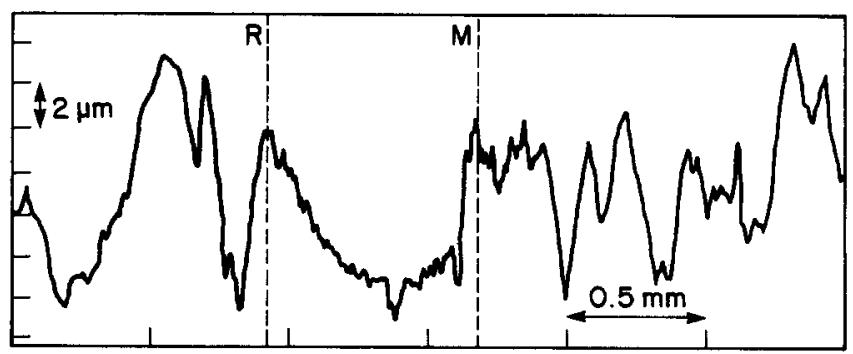

Fig 9 A profile of specimen 6 after experiment $H$, taken perpendicular to the sliding movement; $R M$ represents the wear spot 
smaller than $1 \mu \mathrm{m}$ is effective in reducing the friction coefficient of two titanium alloy surfaces $(f \approx 0.8$ with no coating) throughout the experiment (ie until $\left.n=1.7 \times 10^{6}\right)$.

\section{Conclusions}

It is concluded that Teflon-S coatings effectively reduce friction in $\mathrm{Ti}-6 \mathrm{Al}-4 \mathrm{~V}$ pairs, to be used on the screw and in the polygonal-shaped tube of an extendable prosthesis. The initial layer thickness of the coating affects the coefficient of friction, and in view of its behaviour in time, it is advisable to apply coatings of at least $20 \mu \mathrm{m}$ initial thickness.

\section{References}

1. Krieken F.M. van. Methodical design of a modular femur endoprosthesis. PhD Thesis, University of Twente, Enschede, 1987, ISBN 9071382079

2. Verkerke G.J., Veth R.P.H., Schraffordt Koops H., Kroonenberg H.H. van den, Grootenboer H.J., Nielsen H.K.L. and Oldhoff J. A lengthening element for a modular femur endoprosthetic system. Trans. 35th Ann. Meet., Orth. Res. Soc., Las Vegas, 1989, 494

3. Vos H.B. Wrijving- en slijtageonderzoek aan de groeiprothese. MSc Thesis, Faculty of Mechanical Engineering, University of Twente, Enschede, 1988, (in Dutch)

4. Sass F., Bouché Ch. and Leitner A. Dubbels Taschenbuch für den Maschinenbau, Springer-Verlag, Berlin, 1966

5. Ligterink D.J. Wrijving van met Tefion-S gecoate oppervlakken bij schavielen. Internal report (UT/LGT/1987.8.27), University of Twente, Enschede, 1987, (in Dutch) 